\title{
La Voce dei Pazienti
}

\section{Le cisti rovinano la linea. $\mathrm{E}$ i reni, è meglio toglierli?}

\author{
I pazienti discutono e si scambiano informazioni e opinioni sulla pagina Facebook di AIRP. \\ In questo numero, due tematiche sono in primo piano. Anzitutto, il problema delle cisti che, moltiplicandosi, \\ deformano il girovita e fanno aumentare la pancia, come in una simil-gravidanza. \\ Poi, l'annosa questione se sia meglio tenere i propri reni, anche se malati, \\ oppure farseli asportare ed entrare in dialisi.
}

F.O.: Buongiorno a tutti e grazie per avermi accettata nel gruppo.

Ho 31 anni, valori ancora tutti nella norma, per fortuna, reni, fegato e ovaie policistici e un antipatico fibroma uterino... ogni anno le cisti aumentano e, da due anni a questa parte, è aumentato notevolmente il mio girovita. Ho sentito molti pareri medici, perché mi chiedevo se questo fosse causato da intolleranze, come la celiachia, perché questi cm aumentano nella parte alta dell'addome, e ho pensato potesse anche trattarsi di patologie digestive, pur non avendo sintomi, ma nulla... ora chiedo a voi... soprattutto donne... sembrate anche voi in dolce attesa? È una questione legata alla policistosi? $C$ 'è modo di attenuare questo problema?

Grazie.

A.M.: Cara F.O., hai mai sentito parlare di miracoli? Ecco, per noi non ce ne sono; in compenso, ci teniamo il nostro bel girovita aumentato e, quando ci chiedono se siamo in dolce attesa, noi dolcemente... li mandiamo in attesa in qualche parte del mondo... benvenuta!

D.C.: Confermo questa fatidica lievitazione al girovita... ma la pancia si è sviluppata un paio di anni fa... fino ai 48 anni, pur peggiorando, la pancia non si vedeva... poi sono diventata una focaccia... ma tu sei giovanissima.

A.I.: Però ha i suoi vantaggi: in coda al supermercato mi fanno passare davanti, in metro mi cedono il posto... benvenuta F.O.!

D.C.: Ho chiesto e richiesto se fosse possibile bucare le cisti e aspirarle... ma dicono che poi c'è il rischio di infezione e tendono a dire di NO.

G.N.: Ciao F.O., benvenuta. Se il nostro girovita non si vede più come una volta pazienza, l'importante è stare bene.
R.T.: Io credo di essere una delle poche che, pur essendo policistica, ha i reni di dimensioni quasi normali! Anche per questo, quando ho fatto il trapianto, il nuovo rene è stato aggiunto ai miei!

D.C.: R.T., sei fortunata, allora, e direi rara, perché la policistosi ha come caratteristica l'aumento della massa renale... però buono a sapersi.

R.T.: Infatti me l'hanno detto i nefrologi che per fortuna non sono come mio fratello. I suoi reni, quando ha fatto la nefrectomia, pesavano $4 \mathrm{~kg}$ l'uno! I miei sono aumentati, ma non tantissimo e poi io un po' di pancia l'ho sempre avuta!

F.O.: Che fortunata! Io invece ho sempre avuto una bella pancia piatta. Ora sono aumentata di qualche chilo e l'ho messo tutto lì, sembro proprio incinta. Che disagio... non riesco più a guardarmi allo specchio. Lo so che l'importante è stare bene, ma io non mi riconosco più e il pensiero di essere così per tutta la vita mi fa stare malissimo.

R.T.: Purtroppo la nostra malattia è questa; ci è capitata e non possiamo farci niente, se non imparare a conviverci! Io, oltretutto, sono stata superfortunata perché ho trovato il donatore in mio marito, quindi non ho neanche fatto dialisi!

S.M.: Ciao F.O.! Non preoccuparti, a mia madre sono stati asportati sia il rene destro che il sinistro e insieme pesavano 30 $\mathrm{Kg}$ (un caso eccezionale). Ora è magrissima. Per tutta la vita si è preoccupata del parere della gente, ora invece pensa di aver sprecato solo lacrime e troppo tempo con gente che non capiva i veri problemi di salute. Non dare troppa importanza a tutto questo e vivi bene la tua vita... in bocca al lupo!!!

C.G.: A me hanno fatto tre interventi per "sclerotizzare" un po' di cisti ma ti assicuro che la pancetta sta sempre qui, mia amica fedele... 
M.S.: Io non ho l'addome gonfio, anzi sono piattissima. I miei reni sono di 16 e di $17 \mathrm{~cm}$. Cisti epatiche, ovariche, fibromi uterini. Però i reni mi schiacciano l'intestino e, quindi, ha molte più curve del normale, provocandomi qualche problema.

F.O.: Io comincio a faticare ad allacciarmi le scarpe. L'unico consiglio che ho ricevuto dal nefrologo è di provare a darci dentro con gli addominali, in modo da avere una parete quanto più possibile tonica per contenere l'ingrossamento degli organi.

D.S.: Cara F.O. io ho 39 anni e reni e fegato policistici. Purtroppo, il girovita aumenta e sembro anch'io in dolce attesa, ma ormai non mi fa più né caldo né freddo, l'importante è stare bene. L'unica seccatura è che i tessuti dell'addome si sono allargati e che mi è venuta un'ernia ombelicale che mi fa passare i guai per il dolore! Questa è una conseguenza della nostra malattia! Comunque, ricordati che c'è sempre qualcuno che sta peggio di noi e dobbiamo ritenerci fortunate per questo!

F.O.: Qualcuna di voi ha avuto benefici con l'attività fisica?

M.S.: Benefici? Io ho cominciato a 10 anni a nuotare, ho preso brevetti subacquei e mi sono immersa sino a 5 anni fa; adesso non ho più la forza, però faccio camminate che variano da mezz'ora a un'ora e mezzo. Quando torno, faccio ginnastica a terra: addominali e stretching. Quando, per mancanza di tempo, salto le mie camminate, mi sento "ingolfata". Non so se il fatto di non avere protuberanze e gonfiori è dovuto al movimento. Io ho il ventre e lo stomaco piatti. Ne risente l'intestino che è schiacciato dai reni in un angolino... sicuramente il movimento FA BENE!

F.O.: Io non ho dolori... certo, la pigrizia la fa da padrona!!! Ma se un po' di sacrificio può riportarmi un po' di buonumore, mi impegnerò... grazie ancora per le risposte.

A.M.D.: Ciao a tutti. Vorrei sapere se, per caso, a qualcuno di voi è stato consigliato dal nefrologo di togliere $i$ reni che, pur avendo 2 di creatinina, sono talmente grandi che, tra un anno o due, potrebbero dare gravi problemi. Già a Settembre sono stata ricoverata per un'infezione $e$, per fortuna, sono riuscita a salvare il rene destro con una cura antibiotica molto forte. La mia pancia è grossa quanto quella di una donna al nono mese di gravidanza (quando ho avuto mia figlia non ricordo che fosse cosi grande) e, ovviamente, ho anche il fegato policistico. I medici mi consigliano di togliere $i$ reni e di andare in dialisi e, quando ci sarà un rene disponibile, di fare il trapianto. A qualcuno di voi è stata consigliata la stessa cosa o conoscete qualcuno che ha fatto questo tipo di intervento? Vi ringrazio.

N.C.: Sì, se è il caso; a me, per fortuna, non si è presentata nessuna anomalia, ma, ti ripeto, a me lo hanno detto perché i reni policistici tendono a crescere. Comunque, sono in dialisi peritoneale da 5 anni, piacere di conoscerti.

F.B.: Ciao A.M.D., io ho un'amica a cui da poco sono stati tolti entrambi i reni per le loro enormi dimensioni ( $7 \mathrm{~kg}$ l'uno), anche lei con una pancia enorme; ora la pancia non c'è quasi più ed è in dialisi in attesa del trapianto.

A.M.D.: Ciao N.C., anche per me è un piacere conoscerti. Mia madre, anche lei policistica, ha fatto dialisi per 17 anni, ma senza togliere i reni.

C.G.: Ciao. A mia madre sono stati tolti tutti e due i reni perché anche a lei si erano ingrossati, ma aveva la creatinina più alta. Quello che ti posso dire è che, ovviamente, non potendo più urinare accumulava più acqua, quindi hanno dovuto farle un giorno in più di dialisi. Però, credo che tutto dipenda anche dallo stato di salute generale della persona.

G.B.: Salve, a me è stato consigliato di toglierne almeno uno perché erano grandi... l'ho fatto e non ho avuto problemi né con la dialisi e né dopo il trapianto...

M.G.: Con 2 di creatinina non mi farei operare per togliere entrambi i reni e poi essere costretta ad andare in dialisi. Al massimo ne toglierei uno, farei la domanda di trapianto in anticipo e valuterei se ci possa essere un parente donatore vivente. Dialisi non è bello! Meglio tenersi il pancione...

A.M.: Con 2 di creatinina farei i salti mortali... altro che espianto. Lascia perdere, meglio sembrare in attesa che in dialisi. La questione va affrontata solo se ci sono serie e gravi complicazioni. Auguri.

A.M.D.: A.M., forse non mi sono spiegata; a me non interessa niente della pancia, sono i medici che mi stanno consigliando di togliere i reni, perché dicono che mi possono creare problemi agli altri organi; potrei arrivare al punto di avere difficoltà anche a urinare, visto che le cisti sono talmente grandi che stanno occupando anche la vescica. Avendo 2 di creatinina, sinceramente sono un po' incerta se toglierli, tra l'altro anche il mio fegato è enorme e non escludono il doppio trapianto.

M.G.: Ah, se potessi fare il doppio trapianto, tanto meglio! Una volta ho incontrato due persone che l'hanno fatto e stavano bene. Oggi si fa, ai tempi miei, 17 anni fa, non si faceva. Auguri per tutto, che tu possa trovare la strada giusta!

L.B.: Meglio tenerli i reni fino a quando è possibile. I miei sono grandi $20 \mathrm{~cm}$ ognuno, ma il nefrologo non mi ha detto di toglierli. Mio papà li aveva grandi, ha fatto dialisi e si rompevano spesso le cisti, ma non glieli hanno mai tolti.

A.M.D.: Anche mia madre ha fatto dialisi per 17 anni, anche a lei si rompevano le cisti e aveva spesso ematuria, ma non ha mai tolto i reni. La prima volta che mi si è rotta una cisti mi ha portato un'infezione al rene, ricovero e febbre alta per un mese. 